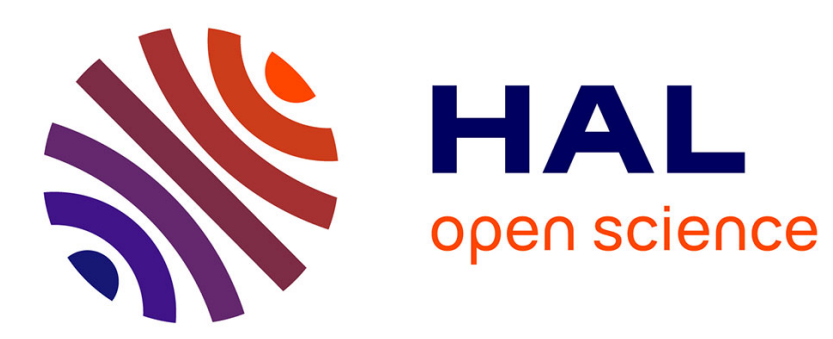

\title{
Backstepping with bounded feedbacks
}

Frederic Mazenc, Abderrahman Iggidr

\section{To cite this version:}

Frederic Mazenc, Abderrahman Iggidr. Backstepping with bounded feedbacks. Systems and Control Letters, 2004, 51 (3-4), pp.235-245. 10.1016/j.sysconle.2003.09.001 . hal-01860796

\section{HAL Id: hal-01860796 https://hal.science/hal-01860796}

Submitted on 30 Aug 2018

HAL is a multi-disciplinary open access archive for the deposit and dissemination of scientific research documents, whether they are published or not. The documents may come from teaching and research institutions in France or abroad, or from public or private research centers.
L'archive ouverte pluridisciplinaire HAL, est destinée au dépôt et à la diffusion de documents scientifiques de niveau recherche, publiés ou non, émanant des établissements d'enseignement et de recherche français ou étrangers, des laboratoires publics ou privés. 


\title{
Backstepping with bounded feedbacks
}

\author{
F. Mazenc, A. Iggidr \\ INRIA Lorraine, Projet CONGE, \\ ISGMP Bât A \\ Ile du Saulcy \\ 57045 Metz Cedex 01, France \\ email: mazenc@loria.fr, Abderrahman.Iggidr@loria.fr
}

\begin{abstract}
An extension of the backstepping approach is proposed. It allows to globally asymptotically stabilize by bounded feedbacks families of nonlinear control systems. Explicit expressions of control laws and Lyapunov functions are given.
\end{abstract}

Key words. Backstepping, bounded feedback, Lyapunov function.

\section{Introduction}

One of the most popular nonlinear techniques of control design is the backstepping approach: see for instance [13, 1] and [4, Chapter 13], where this strategy of design is exposed. The multiple advantages offered by this approach are well-known. Observe in particular that this technique yields a wide family of globally asymptotically stabilizing control laws, allows to address robustness issues and to solve adaptive problems. However, for a long time, it was an accepted fact that this technique could not be used to solve the problem of designing feedbacks bounded in norm, which in many practical situations should be addressed: for instance the possibility of actuator saturation or constraints on actuators impose bounded input and output feedback stabilization problems can be solved only when bounded feedbacks can be determined (see [9]). To quote [10] (page 171), " saturation is probably the most commonly encountered nonlinearity in control engineering ". But it turns out that in fact the backstepping approach can be adapted to the problem of designing bounded feedbacks. In recent work [2], R. Freeman and L. Praly, have shown that for some systems (an $n$-dimensional chain of integrators for instance), bounded stabilizing feedbacks can be constructed by applying a new version of this technique. This result essentially applies to the particular systems in feedback form described by the equations:

$$
\left\{\begin{aligned}
\dot{x} & =f(x)+g(x) z, \\
\dot{z} & =u+h(x, z),
\end{aligned}\right.
$$

where $u \in \Re$ is the input, $x \in \Re^{n_{x}}, z \in \Re, f(0)=0, h(0,0)=0$ and the stabilizing control laws determined are simple and reminiscent of the nested saturation control laws proposed in $[11,12]$.

In the present work, we complement [2] in several directions: 
- We consider systems which admit the following representation:

$$
\left\{\begin{array}{l}
\dot{x}=f(x, z), \\
\dot{z}=u+h(x, z),
\end{array}\right.
$$

where $u \in \Re$ is the input, $x \in \Re^{n_{x}}, z \in \Re, f(0,0)=0, h(0,0)=0$. Observe that the $x$-subsystem of (2) is nonaffine with respect to the variable $z$.

- The Lyapunov functions we construct to prove the global asymptotic stabilizability by bounded feedbacks of the systems (2) are considerably simpler than those proposed in [2]: they belong to the family

$$
U(x, z)=V(x)+\frac{1}{2}\left[\Omega(z)-z_{s}(x)\right]^{2}
$$

where $\Omega(\cdot)$ is a function of class $\mathcal{K}_{\infty}$ and $z_{s}(x)$ is a stabilizing feedback for the $x$-subsystem with $z$ as virtual input.

- The assumptions we impose on the $x$-subsystem of (2) are less restrictive than those given in [2]. In particular, we can carry out the control design even when only a Lyapunov function which is not a strict Lyapunov function ${ }^{1}$ is available for the $x$-subsystem in closed loop with a fictitious feedback $z_{s}(x)$.

- The Lyapunov functions we construct give us a lot of flexibility in the design of the feedbacks. One can construct control laws with shapes very different from the nested saturation control laws presented in [2].

For the sake of clarity, we have chosen to restrict ourselves to the systems (2): the control design can be easily carried out for these systems. However it is worth noting that the key ideas of our approach can be used in several contexts beyond the scope of the present work. In particular, they can be utilized to solve the problem of the construction of bounded feedbacks for systems of the form

$$
\left\{\begin{aligned}
\dot{x} & =f(x, z)+h(x, y, u) u, \\
\dot{z} & =u,
\end{aligned}\right.
$$

which, due to the term $h(x, y, u) u$, are not in feedback form. When some extra assumptions are satisfied, the systems (4) are feedforward systems which can be stabilized using the forwarding approaches exposed for instance in $[8,3,7]$. Interestingly enough even when applied to these systems, the technique of control design of the present work does not coincide with the forwarding approach: for instance, it does not require the determination of the decoupling changes of coordinates used in $[8,7]$ or of the cross terms used in [3], which is an important advantage because in many cases these changes of coordinates or these cross-terms frequently lead to complicated calculations or cannot even be determined due to parameters inaccurately known or the fact that $\dot{x}=f(x, 0)$ is nonlinear.

The paper is organized as follows. In Section 2 two general results are given. The first one is concerned with the case where no strict Lyapunov function for the reduced order system is available and the second with the case where a strict Lyapunov function for the reduced order

\footnotetext{
${ }^{1}$ See the definitions and technical preliminaries for the definitions of Lyapunov function and strict Lyapunov function we adopt.
} 
system is available. Section 3 is devoted to an example. Concluding remarks in Section 4 end the paper.

\section{Definitions and technical preliminaries.}

1. We assume throughout the paper that the functions encountered are sufficiently smooth.

2. The argument of the functions will be omitted whenever no confusion can arise from the context.

3. For a real-valued $C^{1}$ function $k(\cdot)$, we denote by $k^{\prime}(\cdot)$ its first derivative.

4. A positive definite function $V(\cdot)$ on $\Re^{n}$ is a Lyapunov function for the system

$$
\dot{\chi}=\varphi(\chi), \varphi(0)=0
$$

if

$$
\frac{\partial V}{\partial \chi}(\chi) \varphi(\chi) \leq 0, \forall \chi
$$

If besides,

$$
\frac{\partial V}{\partial \chi}(\chi) \varphi(\chi)<0, \forall \chi \neq 0,
$$

then it is a strict Lyapunov function.

5. A real-valued function $k(\cdot)$ is of class $\mathcal{K}_{\infty}$ if it is zero at zero, strictly increasing and becomes unbounded when its argument does.

6. A real-valued function $\sigma(\cdot)$ is a saturation if

- $\sigma(s)$ is nondecreasing.

- $\sigma(s)=\operatorname{sign}(s) \sigma_{M}$ when $|s| \geq 1$ with $\sigma_{M} \in\left[\frac{1}{2}, 1\right]$.

- $\sigma(s)=s$ when $|s| \leq \frac{1}{2}$.

\section{Bounded backstepping: theoretical result}

This section is composed of two parts. In a first part, we propose a set of assumptions which ensure that, for the system (2), globally asymptotically stabilizing bounded feedbacks can be designed when no expression of a strict Lyapunov function for the $x$-subsystem in closed-loop with a stabilizing feedback $z_{s}(x)$ is available. In a second part, we assume that the expression of such a Lyapunov function is available and we exploit it to determine an alternative family of stabilizing bounded feedbacks under a less restrictive assumption on $h(x, y)$.

\subsection{Bounded backstepping without strict Lyapunov function}

We make the following assumptions on (2).

Assumption H1. A function $V(\cdot)$ positive definite, radially unbounded and of class $C^{2}$, a function $W(\cdot)$ nonnegative and of class $C^{1}$ and a control law $z_{s}(x)$ of class $C^{2}$, bounded in norm by $B$ satisfying $z_{s}(0)=0$ and such that

$$
W(x) \leq-\frac{\partial V}{\partial x}(x) f\left(x, z_{s}(x)\right)
$$

are known. Moreover the solution $x(t)=0$ is the unique function satisfying, for all $t$,

$$
\begin{aligned}
\dot{x}(t) & =f\left(x(t), z_{s}(x(t))\right), \\
W(x(t)) & =0 .
\end{aligned}
$$


Assumption H2. There exist two functions $\Gamma_{1}(\cdot)$ and $\Gamma_{2}(\cdot)$ nondecreasing and nonnegative on $[0,+\infty[$ such that

$$
|T(x, z)| \leq \Gamma_{1}(|z|)
$$

with

$$
T(x, z)=\frac{\frac{\partial V}{\partial x}(x)\left[f(x, z)-f\left(x, z_{s}(x)\right)\right]}{z-z_{s}(x)}
$$

and

$$
\left|\frac{\partial z_{s}}{\partial x}(x) f(x, z)\right| \leq \Gamma_{2}(|z|) .
$$

Assumption H3. The function $h(x, z)$ is bounded in norm by a constant $H_{M}$.

Let us state the first result of the work.

Theorem 2.1. Assume Assumptions $H 1$ to H3 are satisfied by the system (2). Then one can construct a function $\Omega(\cdot)$ of class $\mathcal{K}_{\infty}$ and of class $C^{2}$ such that the derivative of the positive definite and radially unbounded function

$$
U(x, z)=V(x)+\frac{1}{2}\left[\Omega(z)-z_{s}(x)\right]^{2}
$$

along the trajectories of the system (2) in closed-loop with

$$
u_{s}(x, z)=-h(x, z)+\frac{1}{\Omega^{\prime}(z)}\left[-\frac{\left(z-z_{s}(x)\right)\left(\rho\left(z-z_{s}(x)\right)+T(x, z)\right)}{\Omega(z)-\Omega\left(z_{s}(x)\right)}+\frac{\partial z_{s}}{\partial x}(x) f(x, z)\right],
$$

where $\rho(\cdot)$ is a a bounded function of class $C^{1}$ and such that $s \rho(s)$ is positive definite, is nonpositive and negative definite if $W(x)$ is positive definite. Moreover, the system (2) in closed-loop with $u_{s}(x, z)$ is globally asymptotically stable and $u_{s}(x, z)$ is bounded in norm.

\section{Discussion of Theorem 2.1.}

- Assumption H1 is a very natural assumption in the framework of the backstepping technique: we require for the $x$-subsystem, with $z$ as virtual input, the knowledge of a globally asymptotically stabilizing feedback and the knowledge of a Lyapunov function which does not necessarily admit a derivative along the trajectories of the closed-loop system which is negative definite. Observe that the existence of a strict Lyapunov function for the system $\dot{x}=f\left(x, z_{s}(x)\right)$ is ensured by the converse Lyapunov theorem (see [5]). But, from a practical point of view, the determination of explicit expressions of strict Lyapunov functions cannot always be carried out or is more complicated than the construction of a Lyapunov function which is not strict. Naturally Assumption $\mathrm{H} 1$ will lead us to prove Theorem 2.1 by invoking the LaSalle Invariance Principle [6].

- The assumptions of Theorem 2.1 do not imply that the system (2) is locally exponentially stabilizable. It follows that Theorem 2.1 applies to feedforward systems which cannot be stabilized through the forwarding designs of [8] or [3]: the three dimensional feedforward system

$$
\left\{\begin{aligned}
\dot{x}_{1} & =x_{2}, \\
\dot{x}_{1} & =-x_{1}^{3}+z^{3}, \\
\dot{z} & =u,
\end{aligned}\right.
$$

with $x=\left(x_{1}, x_{2}\right)^{\top}$, which is not locally exponentially stabilizable, illustrates this fact. 
- Roughly speaking, Assumption H2 is concerned with the growth properties of the functions $V(x), z_{s}(x), f(x, z)$ with respect to the variable $x$. One cannot remove these assumptions without replacing them by other assumptions: the two dimensional system

$$
\left\{\begin{array}{l}
\dot{x}=x^{4} z, \\
\dot{z}=u,
\end{array}\right.
$$

which satisfies Assumptions H1 and H3 does not satisfy Assumption H2 and admits unbounded trajectories whenever $u$ is a function bounded in norm by a constant. The so-called finite escape time phenomenon occurs.

- The function $U(x, z)$ in (13) is only one of the Lyapunov functions whose derivative along the trajectories of the system (2) can be rendered nonpositive by bounded state feedback. In particular, all the functions $V(x)+k\left(\frac{1}{2}\left[\Omega(z)-z_{s}(x)\right]^{2}\right)$ where $k(\cdot)$ is a function zero at zero such that $k^{\prime}(s) \geq 1$ for all $s$ also have this property. The flexibility offered by the function $k(\cdot)$ can be used to address some robustness issues.

Proof of Theorem 2.1. First observe that all the functions (13) where $\Omega(\cdot)$ is a function of class $\mathcal{K}_{\infty}$ are positive definite and radially unbounded.

Next, to simplify the control design we introduce the notation

$$
Z=\Omega(z)-z_{s}(x) .
$$

We impose on $\Omega(\cdot)$ to be a real-valued function such that

$$
\Omega(s)=s, \forall s \in[-2 B, 2 B] \quad, \quad \Omega^{\prime}(s) \geq 1, \forall s .
$$

A simple calculation yields

$$
\dot{Z}=\Omega^{\prime}(z)(u+h(x, z))-\frac{\partial z_{s}}{\partial x}(x) f(x, z) .
$$

On the other hand,

$$
\begin{aligned}
\dot{V} & =\frac{\partial V}{\partial x}(x) f(x, z) \\
& \leq-W(x)+\frac{\partial V}{\partial x}(x)\left[f(x, z)-f\left(x, z_{s}(x)\right)\right] \\
& \leq-W(x)+T(x, z)\left(z-z_{s}(x)\right) .
\end{aligned}
$$

It follows that the derivative of the function

$$
U(x, z)=V(x)+\frac{1}{2} Z^{2}
$$

satisfies the inequality

$$
\dot{U} \leq-W(x)+T(x, z)\left(z-z_{s}(x)\right)+Z\left[\Omega^{\prime}(z) u_{1}-\frac{\partial z_{s}}{\partial x}(x) f(x, z)\right]
$$

with $u_{1}=u+h(x, z)$. The function $\Omega(\cdot)$ is equal to the identity on $[-2 B, 2 B]$ and $z_{s}(x)$ is smaller in norm than $B$. It follows that the equalities

$$
Z=\Omega(z)-z_{s}(x)=\Omega(z)-\Omega\left(z_{s}(x)\right)
$$

are satisfied. Besides, $\Omega^{\prime}(s) \geq 1$ for all $s$ which implies that, for all $x, z$, the inequality

$$
\frac{\Omega(z)-\Omega\left(z_{s}(x)\right)}{z-z_{s}(x)} \geq 1
$$


is satisfied and that the function $\frac{\Omega(z)-\Omega\left(z_{s}(x)\right)}{z-z_{s}(x)}$ is of class $C^{1}$. Using (22) and (23), one obtains

$$
\dot{U} \leq-W(x)+\left[T(x, z)+\frac{\Omega(z)-\Omega\left(z_{s}(x)\right)}{z-z_{s}(x)}\left(\Omega^{\prime}(z) u_{1}-\frac{\partial z_{s}}{\partial x}(x) f(x, z)\right)\right]\left(z-z_{s}(x)\right) .
$$

The feedback (14) yields

$$
\dot{U} \leq-W(x)-\left(z-z_{s}(x)\right) \rho\left(z-z_{s}(x)\right) \leq 0 .
$$

To prove that the origin of the system (2) is globally asymptotically stabilized by the control law defined in (14), we apply the LaSalle Invariance Principle [6]. Let $\left(x_{p}(t), z_{p}(t)\right)$ denote a solution of (2) in closed-loop with the feedback (14) such that

$$
W\left(x_{p}(t)\right)+\left(z_{p}(t)-z_{s}\left(x_{p}(t)\right)\right) \rho\left(z_{p}(t)-z_{s}\left(x_{p}(t)\right)\right)=0 \quad, \quad \forall t \geq 0 .
$$

Then $x_{p}(t)$ and $z_{p}(t)$ satisfy, for all $t \geq 0$,

$$
W\left(x_{p}(t)\right)=0 \quad, \quad z_{p}(t)=z_{s}\left(x_{p}(t)\right)
$$

and

$$
\dot{x}_{p}(t)=f\left(x_{p}(t), z_{s}\left(x_{p}(t)\right)\right) .
$$

According to Assumption H1, it follows that the function $x_{p}(\cdot)$ satisfies

$$
x_{p}(t)=0 \quad, \quad \forall t \geq 0 .
$$

Combining (30) and (28) yields

$$
x_{p}(t)=0 \quad, \quad z_{p}(t)=0 \quad, \quad \forall t \geq 0
$$

which ensures, according to the LaSalle Invariance Principle, that the origin of (2) in closed-loop with (14) is globally asymptotically stable.

The last step of the proof consists in showing that one can determine functions $\rho(\cdot)$ and $\Omega(\cdot)$ such that the corresponding feedback $u_{s}(x, z)$ is bounded.

Since $\Omega^{\prime}(s) \geq 1$ for all $s$, it follows that

$$
\begin{aligned}
\left|u_{s}(x, z)+h(x, z)\right| & \leq \frac{1}{\Omega^{\prime}(z)}\left|\frac{\left(z-z_{s}(x)\right)\left[\rho\left(z-z_{s}(x)\right)+T(x, z)\right]}{\Omega(z)-\Omega\left(z_{s}(x)\right)}-\frac{\partial z_{s}}{\partial x}(x) f(x, z)\right| \\
& \leq \frac{1}{\Omega^{\prime}(z)}\left[\left|\rho\left(z-z_{s}(x)\right)\right|+|T(x, z)|+\left|\frac{\partial z_{s}}{\partial x}(x) f(x, z)\right|\right] .
\end{aligned}
$$

Choosing $\rho(\cdot)$ smaller in norm than a strictly positive real number $\rho_{M}$ and exploiting the inequalities (10) and (12) in Assumption H2, one obtains

$$
\left|u_{s}(x, z)+h(x, z)\right| \leq \frac{1}{\Omega^{\prime}(z)}\left[\rho_{M}+\Gamma_{1}(|z|)+\Gamma_{2}(|z|)\right] .
$$

One can easily determine a function $\Omega(\cdot)$ such that the right-hand-side of this inequality is bounded. A possible choice is

$$
\Omega(s)=s+\int_{0}^{s} \frac{\max \{0,(|l|-2 B)\}(|l|-2 B)\left(\rho_{M}+\Gamma_{1}(|l|)+\Gamma_{2}(|l|)\right)}{4 B^{2}\left[\rho_{M}+\Gamma_{1}(4 B)+\Gamma_{2}(4 B)\right]} d s .
$$

Indeed, for such a choice

$$
\left|u_{s}(x, z)+h(x, z)\right| \leq \frac{\rho_{M}+\Gamma_{1}(|z|)+\Gamma_{2}(|z|)}{1+\frac{\max \{0,(|z|-2 B)\}(|z|-2 B)\left(\rho_{M}+\Gamma_{1}(|z|)+\Gamma_{2}(|z|)\right)}{4 B^{2}\left[\rho_{M}+\Gamma_{1}(4 B)+\Gamma_{2}(4 B)\right]}} .
$$


So, when $|z| \geq 4 B$,

$$
\left|u_{s}(x, z)+h(x, z)\right| \leq \frac{\rho_{M}+\Gamma_{1}(|z|)+\Gamma_{2}(|z|)}{1+\frac{4 B^{2}\left(\rho_{M}+\Gamma_{1}(|z|)+\Gamma_{2}(|z|)\right)}{4 B^{2}\left[\rho_{M}+\Gamma_{1}(4 B)+\Gamma_{2}(4 B)\right]}} \leq \rho_{M}+\Gamma_{1}(4 B)+\Gamma_{2}(4 B)
$$

and, when $|z| \leq 4 B$,

$$
\left|u_{s}(x, z)+h(x, z)\right| \leq \rho_{M}+\Gamma_{1}(4 B)+\Gamma_{2}(4 B) .
$$

It follows readily from Assumption $\mathrm{H} 3$ that, for all $(x, z)$,

$$
\left|u_{s}(x, z)\right| \leq H_{M}+\rho_{M}+\Gamma_{1}(4 B)+\Gamma_{2}(4 B) .
$$

This concludes the proof.

\subsection{Bounded backstepping with strict Lyapunov functions}

Instead of Assumptions $\mathrm{H} 1$ and $\mathrm{H} 3$, we consider now the following assumptions:

Assumption H1'. A function $V(\cdot)$ positive definite, radially unbounded of class $C^{1}$, a function $W(\cdot)$ of class $C^{1}$ and positive definite and control law $z_{s}(x)$ of class $C^{2}$, bounded in norm by $B$, satisfying $z_{s}(0)=0$ and such that

$$
W(x) \leq-\frac{\partial V}{\partial x}(x) f\left(x, z_{s}(x)\right)
$$

are known. Moreover, there exists a positive number $c$ such that

$$
W(x) \geq c|x|^{2}, \forall x:|x| \leq 1 .
$$

Assumption H3'. The function $h(x, z)$ is such that $h(x, z)=h_{1}(x, z)+h_{2}(x, z)$ where $h_{1}(x, z)$ is bounded in norm and there exists a nonnegative function $H(x)$ bounded by a positive real number $H_{M}$ such that

$$
\sup _{|z| \leq\left|z_{s}(x)\right|}\left|h_{2}(x, z)\right| \leq H(x) \leq H_{M}, H(0)=0
$$

and, for all $(x, z)$,

$$
z h_{2}(x, z) \leq 0 .
$$

Let us state the second result of the work.

Theorem 2.2. Assume Assumptions H1', H2 and H3' are satisfied by the system (2). Then one can construct a positive function $\lambda(x)$ and a function $\Omega(\cdot)$ of class $\mathcal{K}_{\infty}$ and of class $C^{2}$ such that the derivative of the positive definite and radially unbounded function

$$
U(x, z)=V(x)+\frac{1}{2}\left[\Omega(z)-z_{s}(x)\right]^{2}
$$

along the trajectories of the system (2) in closed-loop with the bounded feedback

$$
u_{s}(x, z)=-\mu \sigma\left(\lambda(x)\left(\Omega(z)-z_{s}(x)\right)\right)-h_{1}(x, z)
$$

where $\mu$ is a positive real number, where $\sigma(\cdot)$ is a saturation ${ }^{2}$ is negative definite. Moreover, the system (2) in closed-loop with $u_{s}(x, z)$ is globally asymptotically stable.

\footnotetext{
${ }^{2}$ See the definitions and technical preliminaries.
} 
particular case neighborhood family of for large values design.

Remark. The expression feedback (44) is closer to the feedbacks used in [2] than the feedback (14). The difference consists in the presence of the functions $\lambda(x)$ and $\Omega(z)$ in (44) To some extent, Theorem 2.2 establishes a link between the approach of [2] and the one of the present paper.

Remark. One can easily modify the forthcoming proof to show that Theorem 2.2 can be proved by considering the bounded feedbacks $u_{s}(x, z)=-\mu \sigma\left(\lambda(x)\left(\Omega(z)-z_{s}(x)\right)\right)$, instead of the bounded feedbacks (44). It follows that the control design can be carried out even when $h_{1}(x, z)$ is not accurately known.

Proof of Theorem 2.2. Following verbatim the beginning of the proof of Theorem 2.1 we are led to consider, instead of Equality (22), the equality

$$
\begin{aligned}
\dot{U} \leq & -W(x)+T(x, z)\left(z-z_{s}(x)\right)-\mu \Omega^{\prime}(z) Z \sigma(\lambda(x) Z)+\Omega^{\prime}(z) Z h_{2}(x, z) \\
& -Z \frac{\partial z_{s}}{\partial x}(x) f(x, z) .
\end{aligned}
$$

According to Assumption H3, we deduce that

$$
\Omega^{\prime}(z) Z h_{2}(x, z) \leq \Omega^{\prime}(z)|Z| H(x) .
$$

Moreover, Assumption $\mathrm{H} 2$ and the inequality $\Omega^{\prime}(s) \geq 1$, valid for all $s$, ensure that one can determine a positive definite functions $\eta(\cdot)$ independent from $\Omega(\cdot)$, bounded in norm by a positive real number $\eta_{M}$ and such that

$$
\left|T(x, z)\left(z-z_{s}(x)\right)\right|+\left|Z \frac{\partial z_{s}}{\partial x}(x) f(x, z)\right| \leq \eta(|x|) \Gamma(|z|)|Z|
$$

with $\Gamma(s)=\Gamma_{1}(s)+\Gamma_{2}(s)$. We deduce that

$$
\dot{U} \leq-W(x)-\mu \Omega^{\prime}(z) Z \sigma(\lambda(x) Z)+\Omega^{\prime}(z) H(x)|Z|+\eta(|x|) \Gamma(|z|)|Z| .
$$

We distinguish between three cases.

First case. $|\lambda(x) Z| \geq \frac{1}{2}$ and $|z| \geq 2$. Then

$$
\dot{U} \leq-W(x)-\mu \frac{1}{2} \Omega^{\prime}(z)|Z|+\Omega^{\prime}(z) H_{M}|Z|+\eta_{M} \Gamma(|z|)|Z| .
$$

So choosing $\mu \geq \max \left\{10 H_{M}, 10 \eta_{M} \Gamma(3 B)\right\}$ and $\Omega(z)$ suitably yields

$$
\dot{U} \leq-W(x)-\frac{1}{4} \mu \Omega^{\prime}(z)|Z|<0 .
$$

Second case. $|\lambda(x) Z| \geq \frac{1}{2}$ and $|z| \leq 2$. Then

$$
\dot{U} \leq-W(x)-\frac{1}{2} \mu \Omega^{\prime}(z)|Z|+H_{M} \Omega^{\prime}(z)|Z|+\eta_{M} \Gamma(2)|Z|
$$

So choosing $\mu \geq \max \left\{10 H_{M}, 10 \Gamma(3 B), 10 \eta_{M} \Gamma(2)\right\}$ yields

$$
\dot{U} \leq-W(x)-\frac{1}{4} \mu \Omega^{\prime}(z)|Z|<0 .
$$

Third case. $|\lambda(x) Z| \leq \frac{1}{2}$. Then, if we impose on $\lambda(x)$ to be such that $\lambda(x) \geq \frac{1}{B}$ for all $x$, necessarily $|z| \leq \frac{B}{2}+B \leq 2 B$. We deduce that $\Omega^{\prime}(z)=1$ and

$$
\begin{aligned}
\dot{U} & \leq-W(x)-\mu \lambda(x) Z^{2}+H(x)|Z|+\Gamma(2 B) \eta(|x|)|Z| \\
& \leq-W(x)-\frac{\mu}{2} \lambda(x) Z^{2}+\frac{1}{2 \mu \lambda(x)}[H(x)+\Gamma(2 B) \eta(|x|)]^{2} .
\end{aligned}
$$


One can choose $\lambda(\cdot)$ such that $\frac{1}{2 \mu \lambda(x)}[H(x)+\Gamma(2 B) \eta(|x|)]^{2} \leq \frac{1}{2} W(x)$. Such a choice results in

$$
\dot{U} \leq-\frac{1}{2} W(x)-\frac{\mu}{2} \lambda(x) Z^{2} \leq 0 .
$$

The right hand side of this inequality is negative when $(x, z) \neq(0,0)$. This concludes the proof.

\section{Example}

We show the usefulness of Theorem 2.1 by constructing for the three dimensional system

$$
\left\{\begin{aligned}
\dot{x}_{1} & =x_{2} \\
\dot{x}_{2} & =-\frac{x_{1}}{\sqrt{1+x_{1}^{2}}}+\frac{x_{2}}{\sqrt{1+x_{2}^{2}}}+10 z+z^{2}, \\
\dot{z} & =u
\end{aligned}\right.
$$

a globally asymptotically stabilizing bounded state feedback. This system is a good example to illustrate some situations where Theorem 2.1 can be fruitfully utilized: the system (55) is nonaffine with respect to the variable $z$, it is not composed of two passive subsystems and the forwarding approach does not apply to it: the $\left(x_{1}, x_{2}\right)$-subsystem with $z=0$ is not stable. Moreover, for the $\left(x_{1}, x_{2}\right)$-subsystem with $z$ as virtual input, there is a stabilizing feedback which renders nonpositive the derivative along the trajectories of a positive definite and radially unbounded function given by a rather simple expression whereas the expressions of strict Lyapunov functions for this subsystem in closed-loop are more intricate.

First, observe that one can check that Assumptions H1 to H3 are satisfied with $x=$ $\left(x_{1}, x_{2}\right)^{\top}$,

$$
V(x)=\ln \left(\sqrt{1+x_{1}^{2}}+\frac{1}{2} x_{2}^{2}\right)
$$

and

$$
z_{s}(x)=-\frac{1}{5} \frac{x_{2}}{\sqrt{1+x_{2}^{2}}}
$$

According to Theorem 2.1, it follows that the system (55) is stabilizable by bounded feedback. Consequently, by taking advantage of the formula (14), one can determine the expression of a stabilizing feedback bounded in norm for the system (55). However, to illustrate each step of the proof of Theorem 2.1, we carry out the design of a bounded feedback for this system by following this proof step by step.

The derivative of $V(\cdot)$ along the trajectories of (55) satisfies

$$
\begin{aligned}
\dot{V} & =\frac{\frac{x_{2}^{2}}{\sqrt{1+x_{2}^{2}}}+x_{2}\left(10 z+z^{2}\right)}{\sqrt{1+x_{1}^{2}}+\frac{1}{2} x_{2}^{2}} \\
& =\frac{\frac{x_{2}^{2}}{\sqrt{1+x_{2}^{2}}}+10 x_{2} z_{s}(x)+x_{2} z_{s}(x)^{2}+x_{2}\left[10\left(z-z_{s}(x)\right)+z^{2}-z_{s}(x)^{2}\right]}{\sqrt{1+x_{1}^{2}}+\frac{1}{2} x_{2}^{2}} \\
& \leq-\frac{4 x_{2}^{2}}{5 \sqrt{1+x_{2}^{2}}\left(\sqrt{1+x_{1}^{2}}+\frac{1}{2} x_{2}^{2}\right)}+\frac{x_{2}\left[10+z+z_{s}(x)\right]}{\sqrt{1+x_{1}^{2}}+\frac{1}{2} x_{2}^{2}}\left(z-z_{s}(x)\right) .
\end{aligned}
$$


Let $\Omega(\cdot)$ be a function satisfying (18), with $B=\frac{1}{5}$, and consider the variable

$$
Z=\Omega(z)-z_{s}(x)
$$

A simple calculation yields

$$
\dot{Z}=\Omega^{\prime}(z) u+\frac{1}{5\left(1+x_{2}^{2}\right)^{\frac{3}{2}}}\left[-\frac{x_{1}}{\sqrt{1+x_{1}^{2}}}+\frac{x_{2}}{\sqrt{1+x_{2}^{2}}}+10 z+z^{2}\right] .
$$

It follows that the derivative of the function

$$
U(x, z)=V(x)+\frac{1}{2}\left(\Omega(z)-z_{s}(x)\right)^{2}=V(x)+\frac{1}{2} Z^{2}
$$

satisfies the inequality

$$
\begin{aligned}
\dot{U} \leq & -\frac{4 x_{2}^{2}}{5 \sqrt{1+x_{2}^{2}}\left(\sqrt{1+x_{1}^{2}}+\frac{1}{2} x_{2}^{2}\right)}+\frac{x_{2}\left[10+z+z_{s}(x)\right]}{\sqrt{1+x_{1}^{2}}+\frac{1}{2} x_{2}^{2}}\left(z-z_{s}(x)\right) \\
& +Z\left[\Omega^{\prime}(z) u+\frac{1}{5\left(1+x_{2}^{2}\right)^{\frac{3}{2}}}\left(-\frac{x_{1}}{\sqrt{1+x_{1}^{2}}}+\frac{x_{2}}{\sqrt{1+x_{2}^{2}}}+10 z+z^{2}\right)\right] .
\end{aligned}
$$

The feedback

$$
\begin{aligned}
u_{s}(x, z)= & -\frac{z-z_{s}(x)}{\Omega^{\prime}(z)}-\frac{x_{2}\left[10+z+z_{s}(x)\right]}{\sqrt{1+x_{1}^{2}}+\frac{1}{2} x_{2}^{2}} \frac{\left(z-z_{s}(x)\right)}{\left(\Omega(z)-z_{s}(x)\right) \Omega^{\prime}(z)} \\
& +\frac{1}{5 \Omega^{\prime}(z)\left(1+x_{2}^{2}\right)^{\frac{3}{2}}}\left(\frac{x_{1}}{\sqrt{1+x_{1}^{2}}}-\frac{x_{2}}{\sqrt{1+x_{2}^{2}}}-10 z-z^{2}\right)
\end{aligned}
$$

yields

$$
\dot{U} \leq-\frac{4 x_{2}^{2}}{5 \sqrt{1+x_{2}^{2}}\left(\sqrt{1+x_{1}^{2}}+\frac{1}{2} x_{2}^{2}\right)}-\left(\Omega(z)-z_{s}(x)\right)\left(z-z_{s}(x)\right) \leq 0 .
$$

On the other hand, since $\Omega^{\prime}(s) \geq 1$ for all $s$, one can prove that the inequality

$$
\left|u_{s}(x, z)\right| \leq \frac{12}{\Omega^{\prime}(z)}\left(1+z^{2}\right)
$$

is satisfied. We deduce that choosing for instance

$$
\Omega(s)=s+9 \max \left\{0,\left(|s|-\frac{4}{5}\right)^{3}\right\} s
$$

results in a feedback $u_{s}(x, z)$ which satisfies

$$
\left|u_{s}(x, z)\right| \leq 24 .
$$

After lengthy but simple calculations, one obtains the explicit expression of $u_{s}(x, z)$ :

$$
\begin{aligned}
u_{s}(x, z)= & \frac{-5\left(z+\frac{1}{5} \frac{x_{2}}{\sqrt{1+x_{2}^{2}}}\right)\left(1+x_{2}^{2}\right)^{\frac{3}{2}}+\frac{x_{1}}{\sqrt{1+x_{1}^{2}}}-\frac{x_{2}}{\sqrt{1+x_{2}^{2}}}-10 z-z^{2}}{5\left[1+36 \max \left\{0,|z|-\frac{4}{5}\right\}\left(z^{2}-|z|+\frac{4}{25}\right)\right]\left(1+x_{2}^{2}\right)^{\frac{3}{2}}} \\
& -\frac{x_{2}\left(10+z-\frac{1}{5} \frac{x_{2}}{\sqrt{1+x_{2}^{2}}}\right)\left(z+\frac{1}{5} \frac{x_{2}}{\sqrt{1+x_{2}^{2}}}\right)}{\left(\sqrt{1+x_{1}^{2}}+\frac{1}{2} x_{2}^{2}\right)\left(z+9 \max \left\{0,\left(|z|-\frac{4}{5}\right)^{3}\right\} z+\frac{1}{5} \frac{x_{2}}{\sqrt{1+x_{2}^{2}}}\right)\left[1+36 \max \left\{0,|z|-\frac{4}{5}\right\}\left(z^{2}-|z|+\frac{4}{25}\right)\right]}
\end{aligned}
$$




\section{Concluding remarks}

The new design technique proposed does not only complement the backstepping technique. It also shed light on the forwarding approach. On the one hand, from a practical point of view, our main result can be useful even when the forwarding approach can be theoretically applied to (2): this technique relies on changes of coordinates or cross-terms for which it is not always possible to determine explicit formulas when $f(x, 0)$ is not linear and we conjecture that the robustness properties which result from the forwarding approach are significantly different from those which result from our new extension of the backstepping approach. On the other hand, our approach applies, in some cases, to systems which are neither in feedforward nor in feedback form but share features of these two families of nonlinear systems.

A comparative study between the forwarding and the bounded backstepping approach is the subject of future work.

We want to emphasize that the key ideas techniques are even more important than the results themselves. Much remains to be done, robustness and disturbance attenuation issues, discrete-time versions of Theorem 2.1 and Theorem 2.2, are some issues that may be pursued.

Sepulchre and

Acknowledgement. The authors are very indebted to L. Praly and to the anonymous reviewer for their help and comments.

\section{References}

[1] R. Freeman, P. Kokotovic: Robust Nonlinear Control Design. Birkhauser, Boston, 1996.

[2] R. Freeman, L. Praly: Integrators Backstepping for Bounded Controls and Control Rates. IEEE Trans. on Autom. Control, vol. 43, no. 2, pp. 258-262, 1998.

[3] M.J. Jankovic, R. Sepulchre, P.V. Kokotovic: Global stabilization of an enlarged class of cascade nonlinear systems. IEEE Trans. on Autom. Control, vol.41, no.12, pp.1723-1735, 1996.

[4] H. Khalil: Nonlinear Systems. 2nd ed. Prentice Hall, 1996.

[5] J. Kurzweil: On the inversion of Liapunov's second theorem on stability of motion. AMS Trans., Ser. II 24 (1956) 19-77.

[6] J.P. LaSalle: Some extensions of Lyapunov's second method. IRE Trans. Circuit Theory, CT-7(4): 520-527, December 1960.

[7] F. Mazenc: Stabilization of Feedforward Systems Approximated by a Nonlinear Chain of Integrators. Systems \& Control Letters Vol.32, no.4, pp.223-229, 1997.

[8] F. Mazenc, L. Praly: Adding an integration and Global asymptotic stabilization of feedforward systems. IEEE Trans. on Autom. Control, vol.41, no.11, pp.1559-1578, 1996.

[9] F. Mazenc, J.C. Vivalda: Global Asymptotic Output Feedback Stabilization of Feedforward Systems. European Journal of Control (2002)8:1-12.

[10] J-J.E. Slotine, W. Li: Applied Nonlinear Control. Prentice-Hall, Englewood Cliffs, 1991. 
[11] A. Teel: Using saturation to stabilize a class of single-input partially linear composite systems. Proc. IFAC Nonlinear Contr. Syst. Design Symp., Bordeau, France, June 1992.

[12] A. Teel: Feedback stabilization: nonlinear solutions to inherently nonlinear problems. Univ. California, Berkeley, CA. Tech Rep. UCB/ERLM92/65, June 1992.

[13] J. Tsinias: Input to State Stability Properties of Nonlinear Systems and Applications to Bounded Feedback Stabilization Using Saturation. ESAIM: Control, Optimisation and Calculus of Variations. March 1997, Vol. 2, pp. 57-85. 